\title{
Protein Adsorption to Graphene Surfaces Controlled by Chemical Modification of
}

\author{
the Substrate Surfaces
}

\author{
Yasutaka Kamiya, Kenji Yamazaki and Toshio Ogino* \\ Yokohama National University \\ 79-5, Tokiwadai, Hodogaya, Yokohama 240-8501, Japan \\ *E-mail: togino@ynu.ac.jp
}

\begin{abstract}
We have investigated effects of the support substrate surfaces on properties of the attached graphene flakes by observing protein adsorption to the graphene surfaces on $\mathrm{SiO}_{2} / \mathrm{Si}$ substrates that are modified with self-assembled monolayers to control their hydrophilicity. Using atomic force microscopy operated in aqueous environment, we found that high-density clusters of agglomerated avidin molecules form on the graphene flakes in the areas supported by a hydrophobic substrate surface, whereas very low density of large avidin clusters form at the edge of graphene flakes in the area supported by a hydrophilic surface. These results demonstrate that hydrophilicity of the support surface affects hydrophilicity of the graphene surface also in aqueous environment and that surface modification of the support substrate is a useful technique to control protein adsorption phenomena on graphene surfaces for realization of high sensitive graphene biosensors.
\end{abstract}

Keywords: graphene, protein adsorption, biointerfaces, self-assembled monolayers 


\section{Introduction}

Graphene, a 2-dimensional sheet of carbon atoms, has many remarkable properties such as high electron mobility, high mechanical strength and ultimately thin thickness [1], and is expected to be a next-generation material for many device technologies. In device applications, graphene films should be supported with a substrate. Effects of the support substrate surfaces on properties of the attached graphene films have been investigated in air [2,3]. One of the remarkable phenomena related to the substrate is permeable wettability through graphene films that appears as substrate-material dependence of contact angles on the attached single layer graphene (SLG) [4]. It has also been reported that the electrostatic potential of graphene films can be modulated by chemical properties of the support surfaces [5,6]. In spite of intensive research on substrate effects on graphene properties in air, there have been very few reports on them in liquid. Biosensors, including label-free devices $[7,8]$, are one of the promising applications of graphene, and interaction between graphene surfaces and biological molecules in aqueous environment is more important in this target than that in air. Since protein adsorption is strongly influenced by surface characteristics [9,10-12], its dependence on the substrates for graphene support also should be revealed for higher performance of biosensing techniques [13].

In this paper, we investigated protein adsorption to graphene films in aqueous environment focusing on influence of surface modification of the support substrates. We used self-assembled 
monolayers (SAMs) to modify the $\mathrm{SiO}_{2} / \mathrm{Si}$ substrate surfaces because a flat, densely packed monolayer is spontaneously formed on the substrate surface owing to stable chemical bonds to the substrate [14]. In addition, we can easily control the chemical properties of the substrate surfaces, such as their wettability and surface charge, by choosing the functional head groups of SAM molecules. We observed protein adsorption behavior on the graphene films attached to SAM-modified substrates in aqueous environment.

\section{Experimental procedures}

We used silicon (100) substrates with a $300 \mathrm{~nm} \mathrm{SiO}$ layer. The substrates were cleaned by a mixture of sulfuric acid and hydrogen peroxide $\left(\mathrm{H}_{2} \mathrm{SO}_{4}: \mathrm{H}_{2} \mathrm{O}_{2}=3: 1\right)$ at $90{ }^{\circ} \mathrm{C}$ for 10 min and then sonicated for $5 \mathrm{~min}$ in deionized water. SAMs were patterned by the latex beads projection method [15,16]. Polystyrene beads of $2 \mu \mathrm{m}$ in diameter were arrayed on the $\mathrm{SiO}_{2}$ substrates, and a gold layer was deposited by vacuum evaporation. After removal of the polystyrene beads by sonication in deionized water, Au patterns were obtained. Then, a SAM monolayer was formed on the substrates by the vapor phase deposition method. We used two kind of SAMs, octadecyltrichlorosilane (OTS) and 3-aminopropyltriethoxysilane (APTES), both of which were purchased from Sigma-Aldrich. The OTS SAM was formed by heating an OTS solution of $0.5 \mathrm{ml}$ at $100{ }^{\circ} \mathrm{C}$ for 20 hours in a sealed vessel with the substrate. An APTES monolayer was formed by leaving the substrate with an APTES 
solution of $0.5 \mathrm{ml}$ in a sealed vessel at room temperature for 20 hours. The OTS SAM exhibits hydrophobic nature [17] and the APTES SAM hydrophilic one [18]. The used SAMs were bound to the $\mathrm{SiO}_{2}$ surface by stable chemical bonds (silane coupling) in the area where the gold deposition was masked by the polystyrene beads. Since the gold layer was weakly bound to the $\mathrm{SiO}_{2}$ surface, it could be removed by mechanical wiping of the substrates with a paper soaked in acetone. Finally, the substrates were cleaned by sonication in an acetone, ethanol and deionized water for $5 \mathrm{~min}$, respectively.

Graphene flakes were exfoliated from highly oriented pyrolytic graphite (HOPG) using the standard mechanical process [19] and attached to the SAM-modified $\mathrm{SiO}_{2}$ substrates without any chemical treatment. We used avidin from egg white purchased from Nacalai Tesque as the model protein to investigate adsorption behaviors to graphene surfaces. Avidin molecules were dissolved in a buffer solution of N-2-hydroxyethylpiperazine-N'-2-ethanesulfonic acid solution (HEPES, 10mM, $\mathrm{pH}$ 7.0) and its concentration was regulated to be $0.5 \mu \mathrm{g} / \mathrm{ml}$. The substrates were soaked in the avidin solution at room temperature for $30 \mathrm{~min}$. Finally, the avidin solution was replaced by a fresh HEPES solution without containing the avidin molecules to stop further adsorption of avidin molecule.

We observed surface topography and frictional force images of the SAM-modified and graphene-attached substrates by the contact mode of atomic force microscopy (AFM) in air. 
Graphene properties were characterized by Raman spectroscopy. Avidin molecules adsorbed to the substrates were observed by the dynamic force mode of AFM in the buffer solution to prevent conformational change of avidin molecules. Finally, we removed the buffer solution and observed the avidin molecules that remained on the substrate in air using the dynamic force mode.

\section{Results}

Figs. 1(a) and 1(b) show AFM topographic images of an OTS-modified and an APTES-modified $\mathrm{SiO}_{2} / \mathrm{Si}$ substrates in air, respectively. The OTS and APTES monolayers are observed as flat circular patterns of approximately $2 \mu \mathrm{m}$ in diameter, which are in good agreement with polystyrene bead diameter. Figs. 2(a) and 2(b) show AFM topographic and frictional force images of graphene flakes attached to the OTS-modified $\mathrm{SiO}_{2}$ substrate. In Fig. 2(a), a graphene flake is attached to both areas
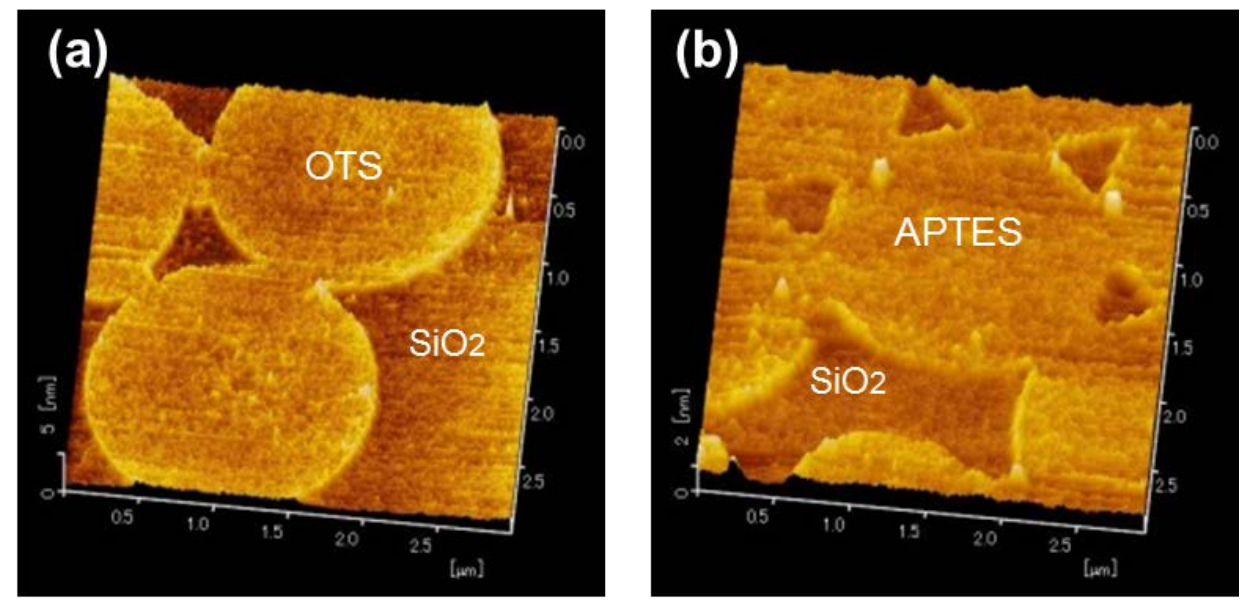

Fig. 1 AFM topographic images of (a) an OTS-modified $\mathrm{SiO}_{2}$ substrate and (b) an APTES-modified one. 
of the OTS-SAM and the $\mathrm{SiO}_{2}$. Height of the graphene surface from the substrate is approximately $0.4 \mathrm{~nm}$, which is similar to the theoretical height of a single layer graphene flake. In Fig. 2(b), frictional force on the graphene surface is smaller than that on the OTS-SAM surface. Since the frictional force in air corresponds to meniscus force between the tip and the surface, the graphene surface is more hydrophobic than that on the OTS-SAM surface though the OTS-SAM surface is more hydrophobic than the $\mathrm{SiO}_{2}$ surface. Figs. 2(c) and 2(d) show AFM topographic and frictional
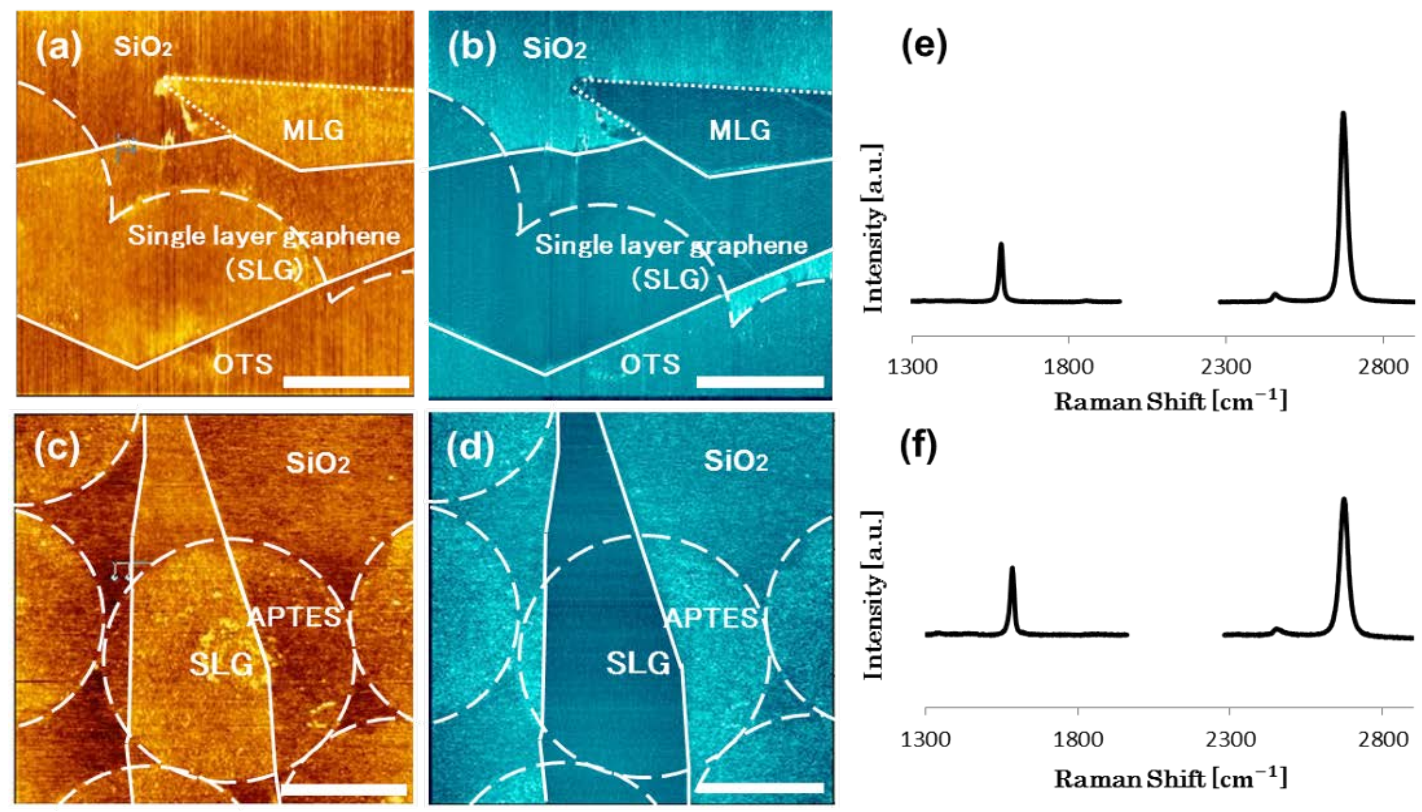

Fig. 2 AFM (a) topographic and (b) frictional force images of the graphene flakes attached to the OTS-modified $\mathrm{SiO}_{2}$ substrate, (c) topographic and (d) frictional force images of the APTES-modified one and corresponding Raman spectra of the graphene flakes attached to (e) the OTS-modified and ( $f$ ) the APTES-modified substrates. The broken white circles indicate the OTS or APTES deposition areas and the white lines the single layer graphene (SLG) areas. The scale bars are 1 $\mu \mathrm{m}$. 
images of a graphene flake attached to the APTES-modified $\mathrm{SiO}_{2}$ substrate. In Fig. 2(c), graphene film is also attached to both the APTES-SAM and the $\mathrm{SiO}_{2}$ areas. In Fig. 2(d), the frictional force on the APTES-SAM surface is much larger than that on the graphene surface and slightly larger than that on the $\mathrm{SiO}_{2}$ surface. It means that the APTES-SAM surface is more hydrophilic than the $\mathrm{SiO}_{2}$ surface. Figs. 2e and 2f show Raman spectra of the graphene flakes that correspond to Figs. 2(a) and 2(b). Layer number of the graphene flakes is important in this study because influence of the backside state of the graphene flakes is expected to appear only when the layer number is small [4]. It can be estimated by an intensity ratio of the $2 \mathrm{D}-\left(\mathrm{I}_{2 \mathrm{D}}\right)$ and $\mathrm{G}-\left(\mathrm{I}_{\mathrm{G}}\right)$ peaks, $\mathrm{I}_{2 \mathrm{D}} / \mathrm{I}_{\mathrm{G}}$, and Figs. 2(e) and
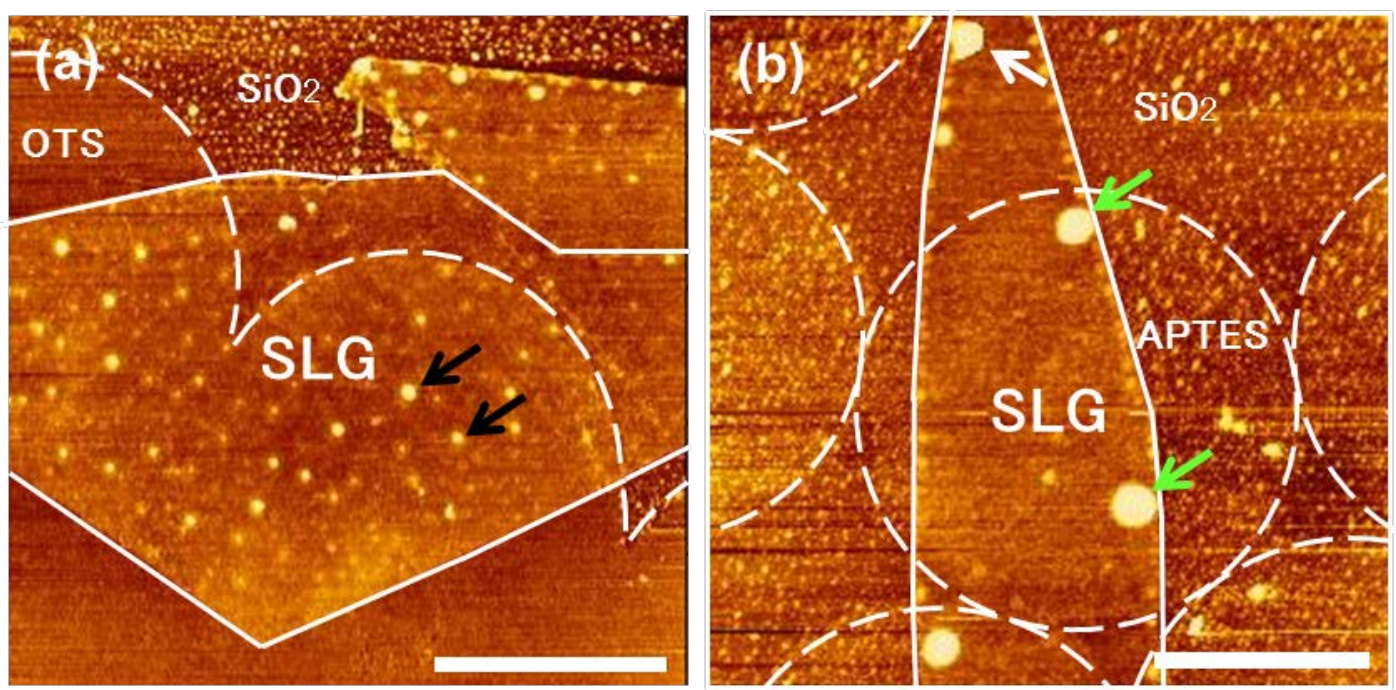

Fig. 3 AFM topographic images of avidin molecules on the graphene flakes attached to (a) the OTS-modified $\mathrm{SiO}_{2}$ substrate and (b) the APTES-modified one. The scale bars are $1 \mu \mathrm{m}$. 
2(f) clearly indicate that single layer graphene flakes are attached to both the OTS-modified $\mathrm{SiO}_{2}$ substrate and the APTES-modified one because the observed values of $\mathrm{I}_{2 \mathrm{D}} / \mathrm{I}_{\mathrm{G}}$ are more than 2 [20].

We investigated effects of hydrophilicity of the support substrate surfaces on protein adsorption to the graphene flakes attached to the SAM-deposited $\mathrm{SiO}_{2}$ surfaces. Fig. 3(a) shows an AFM topographic image of the avidin-adsorbed graphene flake attached to the OTS-modified $\mathrm{SiO}_{2}$ substrate. The $\mathrm{SiO}_{2}$ area without an OTS-SAM is granular, indicating that isolated avidin molecules are adsorbed, but the OTS-SAM areas are apparently flat, indicating that no avidin molecule or denatured molecules are adsorbed to the OTS-SAM surface. The latter case occurs when avidin molecules change their conformation to a flat structure by tight adsorption through a hydrophobic interaction with the OTS-SAM surface. Therefore, the apparently flat OTS surface after avidin adsorption is possibly caused by a denatured avidin layer. On the graphene flake surface, avidin molecules, indicated by the black arrows, preferentially adsorb to the OTS-supported graphene areas compared with the $\mathrm{SiO}_{2}$-supported one. The size of the adsorbed avidin grains on the OTS-supported graphene areas is much larger than that of the single avidin molecule. Agglomerated avidin molecules formed clusters in the OTS-supported graphene areas. Fig. 3(b) shows an AFM topographic image of an avidin-adsorbed graphene flake attached to the APTES-modified $\mathrm{SiO}_{2}$ substrate. We can observe isolated avidin molecules in the $\mathrm{SiO}_{2}$ and the APTES-SAM areas without a graphene flake. On the graphene flake surface, large agglomerated clusters indicated by the green 
arrows are observed only near the edges of the graphene flake. The adsorption behavior of avidin

molecules on the APTES-supported graphene areas is similar to that on the $\mathrm{SiO}_{2}$-supported areas.

We observed avidin molecules attached to the substrates in air after removal of the buffer solution and drying by $\mathrm{N}_{2}$ blow. Figs. 4(a) and 4(b) show AFM topographic images of avidin molecules that remained on the graphene flakes attached to the OTS-modified $\mathrm{SiO}_{2}$ substrate and the
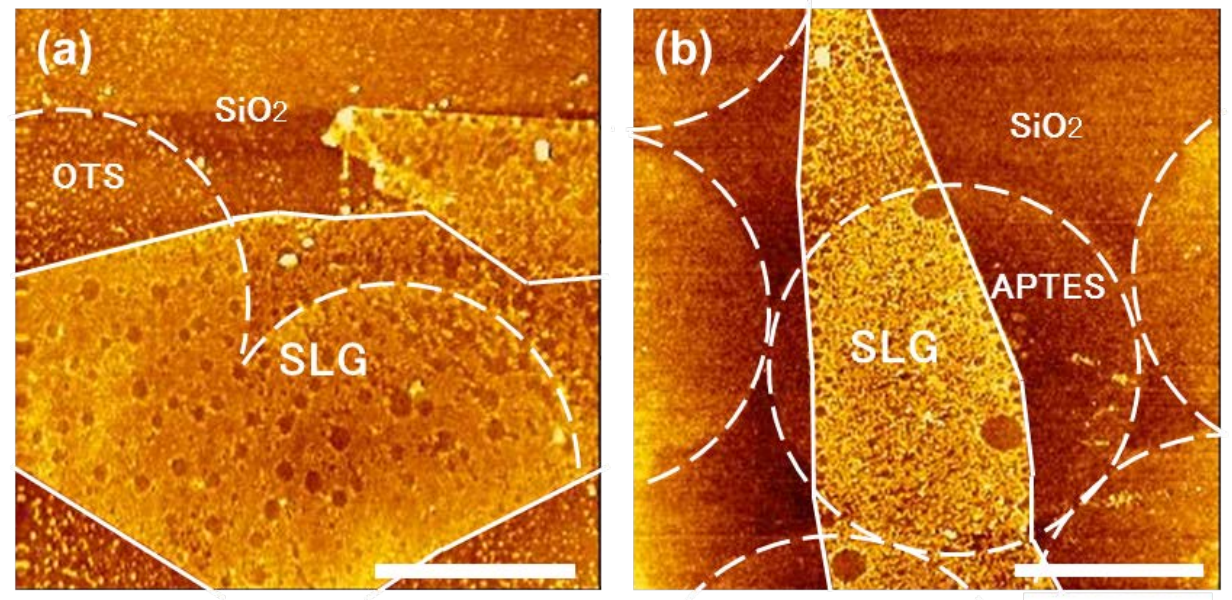

(c)

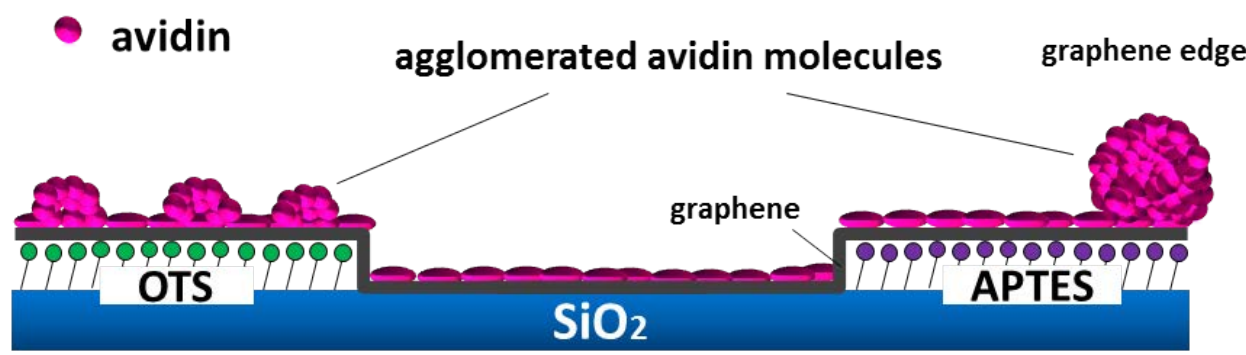

Fig. 4 AFM topographic images of (a) the OTS-modified $\mathrm{SiO}_{2}$ substrate and (b) the APTES-modified one in air, where avidin molecules were adsorbed to those surfaces and then partially removed in the drying process. (c) Sketch of the avidin adsorption in buffer solution. The scale bars are $1 \mu \mathrm{m}$. 
APTES-modified one, respectively, after the drying process. On both substrates, a large number of holes were observed on the whole area of the graphene surfaces. This means that the avidin molecule clusters were detached from the graphene surface. Therefore, the entire graphene surfaces, which were apparently flat before detachment of the clusters as shown in Figs. 3(a) and 3(b), were covered with the layers of deformed avidin molecules in the buffer solutions. Fig. 4(c) shows the sketch of avidin adsorption in the buffer solution. On the OTS-supported graphene surface, a flatten avidin molecule layer and the agglomerated avidin molecule clusters formed. On the $\mathrm{SiO}_{2}$ - and APTES-supported graphene surfaces, only flattened avidin molecule layer covers the graphene surface except the edges of the graphene flake.

\section{Discussions}

First, we discuss avidin adsorption behavior on the graphene flake surfaces shown in Fig. 3. In our results, a high density of agglomerated avidin molecule clusters, indicated by the black arrows in Fig. 3a, formed in the OTS-supported graphene areas, whereas a low density of large clusters formed only in the graphene edge areas on the $\mathrm{SiO}_{2}$ - and APTES-supported surfaces, as indicated by the white arrow and the green arrows, respectively. Since a large avidin cluster is observed on the $\mathrm{SiO}_{2}$-supported surface, as indicated by the white arrow in Fig. 3(b), similar clusters should exist at the graphene edge in the $\mathrm{SiO}_{2}$-supported areas also on the OTS samples though it is not observed in Fig. 3(a). In general, agglomerated protein molecules tend to form on a hydrophobic surface rather 
than a hydrophilic surface [21,22]. When protein molecules adsorb to the hydrophobic surface, they are strongly fixed by the hydrophobic interaction, and their conformation is changed, resulting in a matrix structure where protein molecules interact strongly with each other [23]. The present results suggest that the OTS-supported graphene surface is more hydrophobic than that of the $\mathrm{SiO}_{2}$-supported ones in spite of the same graphene surface.

Next, we discuss effects of the support surface on avidin adsorption to the graphene surface. Obviously, hydrophilicity of the graphene flake surfaces is controlled by their support substrate surfaces. It has been reported that single layer graphene exhibits permeable wetting behavior of the support substrate in air [4]. The present results suggest that a similar phenomenon occurs also in the aqueous environment as well as in air. According to a previous paper, confined water layers exist between the graphene film and the support sapphire substrate in the liquid environment [24]. In air, water molecules are confined at the graphene-substrate interface [25] and the height between the graphene surface and the substrate increases when exposed to air with high humidity at the graphene flake deposition [26]. Therefore, water layers confined at the graphene-substrate interface should be considered to reveal the mechanism of protein adsorption to the graphene surfaces. In air, structure of the water layer on a solid surface depends on hydrophilicity of the surface. In hydrophilic surface, water molecules form a stable layer on the solid surface through site-specific interaction between water molecules and the surface [27]. On the other hand, on hydrophobic solid surfaces, water 
molecules cannot form a stable water layer. This is because attractive interaction between the substrate surface and water molecules is not enough strong compared to water-water interaction. In the present experiments, however, the samples were placed in aqueous environment, and the amount of water molecules on the graphene back surface is large enough for layered structure formation. In this situation, a structured, or ice-like, water layer forms near the solid surface [28]. Since the structure of water layers confined between the graphene flakes and the support substrate depends on both the graphene surface and the substrate surface modified with OTS, APTES or $\mathrm{SiO}_{2}$, properties of the graphene flake surfaces are also influenced by the substrate modification through the interfacial water structure. The structure of the interfacial water layer is not so simple because it should be affected not only by the support substrate surface but also by the attached graphene flakes. Nevertheless, considering the differences in avidin adsorption to the graphene flakes, which are originally the same material, we can conclude that hydrophilicity of the support surface affects avidin adsorption behaviors.

Then, we discuss configuration of the agglomerated avidin clusters on the graphene flake surfaces. In our results, agglomerated high density avidin clusters formed on the OTS-supported graphene areas, while very low-density, much larger clusters formed only near the graphene edges in the $\mathrm{SiO}_{2}$ and APTES-supported areas. It should be considered that degree of agglomeration depends on the mobility of the molecules on the graphene surface. It has been reported that protein molecules 
diffuse on hydrophobic surfaces more slowly than on hydrophilic surfaces because the interaction between protein molecules and the surface is weaker on the hydrophilic surface than the hydrophobic one [29]. Avidin molecules seem to have low mobility in the OTS-supported graphene areas, which are more hydrophobic. Therefore, high-density agglomerated avidin clusters formed in the OTS-supported graphene areas owing to a strong hydrophobic interaction between avidin molecules and the graphene flake surface. On the other hand, avidin molecules seem to have high mobility on the $\mathrm{SiO}_{2}$ - and APTES-supported graphene surfaces, whose hydrophobicity is weak. Thus, large agglomerated avidin clusters formed near the graphene flake edges in the $\mathrm{SiO}_{2-}$ and APTES-supported areas because the interaction between avidin molecules and those surface areas is enough weak except their reactive edges. Fig. 4(c) shows the model of avidin adsorption behavior on graphene films attached to hydrophobic and hydrophilic support substrate surfaces.

Finally, we discuss the denature layer of the avidin molecules. Obviously, holes in the denature layers are observed where the large clusters in the hydrophilic areas or small clusters in the OTS supported areas had existed before removal of the buffer solution by $\mathrm{N}_{2}$ blow. This suggests that the avidin clusters formed in the early stage of the adsorption and then denature layers formed in the late stage. This is probable because after the denature layers have once covered the graphene flake surface, surface diffusion of the avidin molecules would be suppressed by the protein-protein interaction, which is much larger than protein-graphene ones, as suggested by the cluster formation. 
Therefore, we can conclude that avidin adsorption occurs through two stages, diffusion and agglomeration of avidin molecules in the early stage and denature layer formation in the late one.

Generally, protein adsorption to a solid surface caused by a hydrophobic interaction is accompanied with conformational change of protein molecules. Since a graphene surface is strongly hydrophobic, conformational change and spreading of adsorbed protein are expected to happen, as indicated by molecular dynamic simulation [30]. Previously, we suggested that degree of denature of avidin molecules in the adsorption to sapphire surfaces is smaller than that of ferritin molecules. [31]. In the present results, avidin molecules were tightly adsorbed to graphene surfaces owing to the high hydrophobicity of graphene compared with sapphire. However, it was also reported that conformational change is enhanced by AFM observation because an AFM tip forces the protein [30]. Therefore, conformational change should be investigated using additional analytical tools, such as labeling with biotin.

\section{Conclusion}

We observed protein adsorption behavior on the graphene flakes attached to partially SAM-modified $\mathrm{SiO}_{2} / \mathrm{Si}$ substrates in aqueous environment. It was found that a high-density of agglomerated avidin molecule clusters formed in the OTS-supported graphene areas, whereas a low-density of large clusters formed in the $\mathrm{SiO}_{2}$ - and APTES-supported ones, indicating that avidin 
molecule adsorption and its cluster formation are controlled by hydrophilicity of the support surfaces.

This means that hydrophilicity of the support surface affects hydrophilicity of the graphene flake surface also in aqueous environment. The present technique to control protein adsorption phenomena on graphene surfaces can be applied to high performance graphene biosensors with small non-specific adsorption of protein molecules.

Acknowledgements

This work was partly supported by Grant-in-Aid for Scientific Research from the Ministry of Education, Culture, Sports, Science and Technology. 


\section{References}

[1] A. K. Geim, Science 324 (2009) 1530.

[2] T. Tsukamoto, K. Yamazaki, H. Komurasaki, T. Ogino, J. Phys. Chem. C 116 (2012) 4732.

[3] T. Kase, Toshio Ogino, J. Phys. Chem. C 117 (2013) 15991.

[4] J. Rafiee, X. Mi, H. Gullapalli, A. V. Thomas, F. Yavari1, Y. Shi, P. M. Ajayan, N. A. Koratkar, Nat. Mater. 11 (2012) 217.

[5] K. Yokota, K. Takai, T. Enoki, Nano Lett. 11 (2011) 3669.

[6] W. H. Lee, J. Park, Y. Kim, K. S. Kim, B. H. Hong, K. Cho, Adv. Mater. 23 (2011) 3460.

[7] S. Okamoto, Y. Ohno, K. Maehashi, K. Inoue, K. Matsumoto, Jpn. J. Appl. Phys. 51 (2012) 06FD08.

[8] R. Stine, S. P. Mulvaney, J. T. Robinson, C. R. Tamanaha, P. E. Sheehan, Anal. Chem. 85 (2013) 509.

[9] Y. L. Jeyachandran, T. Weber, A. Terfort, M. Zharnikov, J. Phys. Chem. C 117 (2013) 5824.

[10] C. Y. Xue, K. L. Yang, J. Colloid Interface Sci. 344 (2010) 48.

[11] S. Arumugam, V. V. Popik, J. Am. Chem. Soc. 133 (2011) 15730.

[12] O. E. Zubir, I. Barlow, E. Ul-Haq, H. A. Tajuddin, N. H. Williams, G. J. Leggett, Langmuir 29 (2013) 1083.

[13] T. Alava, J. A. Mann, C. Théodore, J. J. Benitez, W. R. Dichtel, J. M. Parpia, H. G. Craighead, Anal. Chem. 85 (2013) 2754.

[14] S. Y. Chen, P. H. Ho, R. J. Shiue, C. W. Chen, W. H. Wang, Nano Lett. 12 (2012) 964.

[15] N. Herzer, S. Hoeppener, U. S. Schubert, H. Fuchs, U. C. Fischer, Adv. Mater. 20 (2008) 346.

[16] J. Yu, C. Geng, L. Zheng, Z. Ma, T. Tan, X. Wang, Q. Yan, D. Shen, Langmuir 28 (2012) 12681.

[17] J. Dong, A. Wang, K. Y. Ng, G. Mao, Thin Solid Films 515 (2006) 2116.

[18] J. A. Howarter, J. P. Youngblood, Langmuir 22 (2006) 11142. 
[19] K. S. Novoselov, A. K. Geim, S. V. Morozov, D. Jiang, Y. Zhang, S. V. Dubonos, I. V. Grigorieva, A. A. Firsov, Science 306 (2004) 666.

[20] A. A. Balandin, S. Ghosh, W. Bao, I. Calizo, D. Teweldebrhan, F. Miao, C. N. Lau, Nano Lett. 8 (2008) 902.

[21] M. Rabe, D. Verdes, S. Seeger, Soft Matter 5 (2009) 1039.

[22] C. C. Dupont-Gillain, C. M. J. Fauroux, D. C. J. Gardner, G. J. Leggett, J. Biomed. Mater. Res. A 67 (2003) 548.

[23] M. Holmberg, X. Hou, Langmuir 25 (2009) 2081.

[24] K. Yamazaki, S. Kunii, T. Ogino, J. Phys. Chem. C 117 (2013) 18913.

[25] K. Xu, P. Cao, J. R. Heath, Science 329 (2010) 1188.

[26] H. Komurasaki, T. Tsukamoto, K. Yamazaki, T. Ogino, J. Phys. Chem. C 116 (2012) 10084.

[27] K. Kobayashi, N. Oyabu, K. Kimura, S. Ido, K. Suzuki, T. Imai, K. Tagami, M. Tsukada, H. Yamada, J. Chem. Phys. 138 (2013) 184704.

[28] K. Suzuki, N. Oyabu, K. Kobayashi, K. Matsushige, H. Yamada, Appl. Phys. Express 4 (2011) 125102

[29] E. P. Vieira, S. Rocha, M. C. Pereira, H. Mohwald, M. A. N. Coelho, Langmuir 25 (2009) 9879.

[30] C. Mücksch, H. M. Urbassek, Langmuir 27 (2011) 12938.

[31] K. Yamazaki, T. Ikeda, T. Isono, T. Ogino, J. Colloid Interface Sci. 361 (2011) 64. 
Figure captions

Fig. 1 AFM topographic images of (a) an OTS-modified $\mathrm{SiO}_{2}$ substrate and (b) an APTES-modified one.

Fig. 2 AFM (a) topographic and (b) frictional force images of the graphene flakes attached to the OTS-modified $\mathrm{SiO}_{2}$ substrate, (c) topographic and (d) frictional force images of the APTES-modified one and corresponding Raman spectra of the graphene flakes attached to (e) the OTS-modified and (f) the APTES-modified substrates. The broken white circles indicate the OTS or APTES deposition areas and the white lines the single layer graphene (SLG) areas. The scale bars are $1 \mu \mathrm{m}$.

Fig. 3 AFM topographic images of avidin molecules on the graphene flakes attached to (a) the OTS-modified $\mathrm{SiO}_{2}$ substrate and (b) the APTES-modified one. The scale bars are $1 \mu \mathrm{m}$. Fig. 4 AFM topographic images of (a) the OTS-modified $\mathrm{SiO}_{2}$ substrate and (b) the APTES-modified one in air, where avidin molecules were adsorbed to those surfaces and then partially removed in the drying process. (c) Sketch of the avidin adsorption in buffer solution. The scale bars are $1 \mu \mathrm{m}$. 